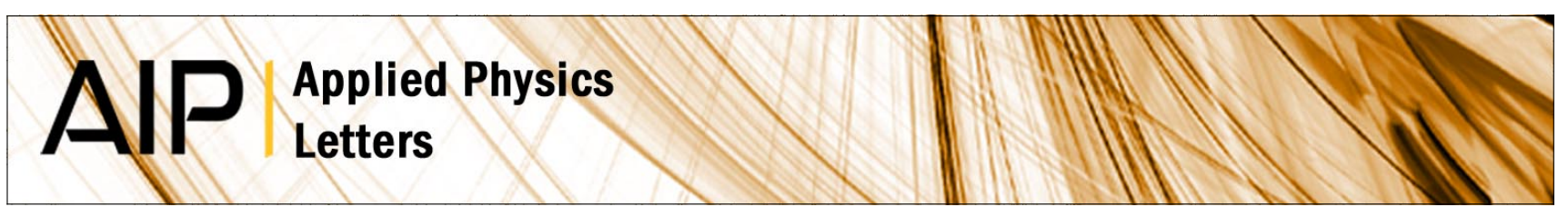

\title{
Dopants effect on the band structure of PbTe thermoelectric material
}

Y. Takagiwa, Y. Pei, G. Pomrehn, and G. J. Snyder

Citation: Appl. Phys. Lett. 101, 092102 (2012); doi: 10.1063/1.4748363

View online: http://dx.doi.org/10.1063/1.4748363

View Table of Contents: http://apl.aip.org/resource/1/APPLAB/v101/i9

Published by the American Institute of Physics.

\section{Related Articles}

First principles calculation of dopant solution energy in $\mathrm{HfO} 2$ polymorphs

J. Appl. Phys. 112, 084514 (2012)

Spectroscopy of 3, 4, 9, 10-perylenetetracarboxylic dianhydride (PTCDA) attached to rare gas samples: Clusters vs. bulk matrices. Il. Fluorescence emission spectroscopy

J. Chem. Phys. 137, 164302 (2012)

Structural transitions and unusual magnetic behavior in Mn-doped $\mathrm{Bi} 1-\mathrm{xLaxFeO} 3$ perovskites

J. Appl. Phys. 112, 084102 (2012)

In-situ synchrotron energy-dispersive x-ray diffraction study of thin Pd foils with $\mathrm{Pd}: \mathrm{D}$ and $\mathrm{Pd}: \mathrm{H}$ concentrations up to $1: 1$

J. Appl. Phys. 112, 083510 (2012)

Atomic-scaled investigation of structure-dependent luminescence in Sialon:Ce phosphors

Appl. Phys. Lett. 101, 161904 (2012)

\section{Additional information on Appl. Phys. Lett.}

Journal Homepage: http://apl.aip.org/

Journal Information: http://apl.aip.org/about/about_the_journal

Top downloads: http://apl.aip.org/features/most_downloaded

Information for Authors: http://apl.aip.org/authors

\section{ADVERTISEMENT}
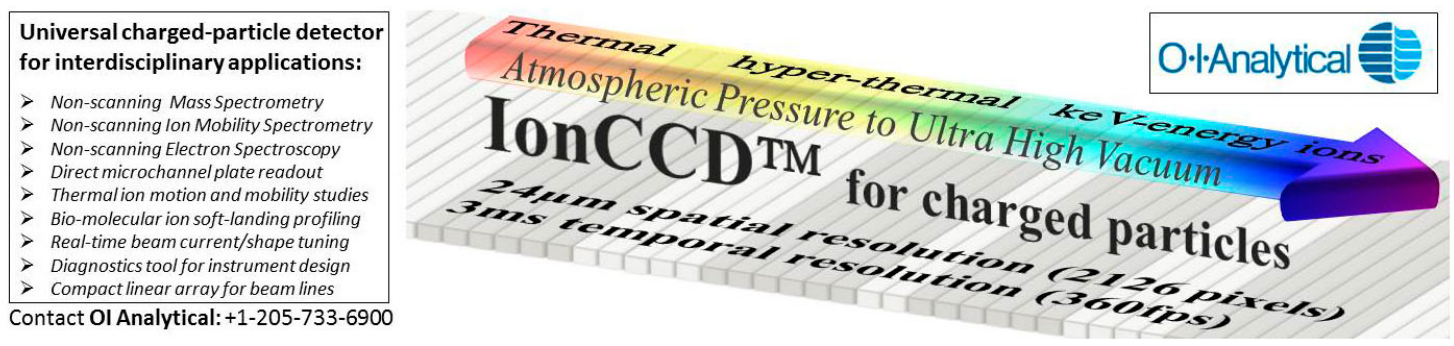


\title{
Dopants effect on the band structure of PbTe thermoelectric material
}

\author{
Y. Takagiwa, ${ }^{1,2, a), b)}$ Y. Pei, ${ }^{2, b)}$ G. Pomrehn, ${ }^{2}$ and G. J. Snyder ${ }^{2}$ \\ ${ }^{1}$ Department of Advanced Materials Science, The University of Tokyo, Kiban-toh 502, 5-1-5 Kashiwanoha, \\ Kashiwa-shi, Chiba 277-8561, Japan \\ ${ }^{2}$ Department of Materials Science, California Institute of Technology, Pasadena, California 91125, USA
}

(Received 29 June 2012; accepted 14 August 2012; published online 27 August 2012)

\begin{abstract}
$\mathrm{PbTe}$ is a promising thermoelectric material and its dimensionless figure of merit, $z T$, can be enhanced by optimizing the band structure near the Fermi level via chemical doping. This letter describes the dopants effect on bandgap, $E_{\mathrm{g}}$, and effective mass, $m^{*}$, for disordered La- and I-doping, based on theoretical calculations. $E_{\mathrm{g}}$ increases with increasing La and decreases with increasing I concentration. While $m^{*}$ increases upon La-doping, I-doping does not change $m^{*}$ noticeably. The calculated results are qualitatively consistent with the experimental results and explain the higher $z T$, up to 1.4 at $800 \mathrm{~K}$, observed in I-doping PbTe compared to La-doping. (C) 2012 American Institute of Physics. [http://dx.doi.org/10.1063/1.4748363]
\end{abstract}

To achieve global energy sustainability, thermoelectric materials, which can generate power through the direct conversion of thermal energy into electrical energy, are attractive because they can contribute to solve the global environmental issues in terms of saving electricity and waste-heat recovery. Thermoelectric materials are evaluated by the dimensionless figure of merit, defined as $z T=S^{2} \sigma T /$ $\left(\kappa_{e l}+\kappa_{p h}\right)$, where $S, \sigma, \kappa_{e l}, \kappa_{p h}$, and $T$ are the Seebeck coefficient, electrical conductivity, electronic thermal conductivity, phonon thermal conductivity, and temperature, respectively. ${ }^{1}$ For the practical application of thermoelectric materials, $z T$ is desired to be above at least unity. From the equation of $z T$, one can easily understand that $S$ and $\sigma$ should be large while $\kappa\left(=\kappa_{e l}+\kappa_{p h}\right)$ should be low to attain a high $z T$ value. There are two primary strategies to improve $z T$; one is optimizing the electronic terms, that is, $S^{2} \sigma / \kappa_{e l}$, which is mainly governed by the local electronic structure (the density of states (DOS), group velocity, and relaxation time) near the Fermi level, $E_{F}{ }^{2}$ The other attempt is lowering $\kappa_{p h}$ by introducing phonon scattering centers such as mass contrast $^{3}$ and nanostructure ${ }^{4}$ in bulk materials.

Doped-lead telluride with NaCl-type crystal structure is a semiconductor material well suited for thermoelectric applications because its $z T$ can be above unity ${ }^{5,6}$ and the band structure is altered by introducing resonant impurity levels near $E_{F}$ (Refs. 7 and 8) or by band structure engineering $^{9-15}$ for optimizing its electronic properties. Since $\kappa_{p h}$ is close to the lower amorphous limit, ${ }^{16}$ due to alloying and nanostructuring with small ${ }^{4,17}$ and large nanostructures, ${ }^{18}$ it is most important to control the band structure near $E_{F}$ via chemical doping for further enhancement of $z T$. To the first order, thermoelectric semiconductors can be approximated by a rigid band structure. ${ }^{19,20}$ However, higher order effects of the chemical doping on the thermoelectric properties have been observed. ${ }^{13,18,21}$ Band structure calculations can provide detailed information to elucidate even small doping

\footnotetext{
a) Author to whom correspondence should be addressed. Electronic mail: takagiwa@phys.mm.t.u-tokyo.ac.jp. Tel/Fax: +81-4-7136-3759.

b) Authors who have contributed equally to this work.
}

effects on the electronic band structure. ${ }^{22}$ The conduction band in PbTe is known to be at the $L$ point. ${ }^{23}$

It has been found that substitution on the $\mathrm{Pb}$ site leads to a lower carrier mobility, $\mu$, while substitution on the Te site has a minor effect. ${ }^{24}$ Alekseeva et al. have investigated the effects of rare-earth impurities on the low temperature $(<300 \mathrm{~K})$ transport properties in $\mathrm{PbTe}$ and indicated that the physical origin could be interpreted by the existence of impurity states in the conduction band. ${ }^{25}$ In this case, the effective mass, $m^{*}$, might be increased by the substitution on the $\mathrm{Pb}$ site. Very recently, Pei et al. have demonstrated that low effective mass leads to high thermoelectric performance on the basis of experimental results of I- or La-doped PbTe based on the acoustic scattering theory. ${ }^{15}$ Their maximum $z T$ exhibit high values of 1.4 and 1.2 for I- and La-doped PbTe, respectively. ${ }^{15}$ Experimentally obtained density of states effective mass of I-doped samples near the optimal carrier concentration is $20 \%$ lower than that of La-doped ones, which leads to $20 \%$ higher $z T$. Even though this difference in effective mass is small, it provides an excellent example of the doping effect beyond the rigid band approximation. Therefore, it is worth investigating the electronic properties, such as bandgap, $E_{\mathrm{g}}$, and effective mass, $m^{*}$, with theoretical calculations, to understand and compare to experimental results in doped materials.

Inspired by the previous experimental results of doped $\mathrm{PbTe},{ }^{15,24,25}$ we investigated the effect of La- or I-doping on the electronic DOS for PbTe semiconductor material. We performed calculations using the Korringa-Kohn-Rostoker Green function formalism under the coherent potential approximation (KKR-CPA $)^{26-28}$ for randomly disordered $\mathrm{La}_{x} \mathrm{~Pb}_{1-x} \mathrm{Te}$ and $\mathrm{PbTe}_{1-x} \mathrm{I}_{x}$. KKR-CPA calculations have been applied in PbTe-systems, ${ }^{8,22,29}$ but there is no report on the comparison with $E_{\mathrm{g}}$ and $m^{*}$ for different $n$-type dopants in PbTe. Here, we focus on the changes of $E_{\mathrm{g}}$ and $m^{*}$ by observing changes in DOS with changing doping concentration.

In all calculations, the room-temperature lattice constant of $6.462 \AA$ (Ref. 30) (12.22 bohr) and the von Barth-Hedin formula $^{31}$ for the exchange energy was used. For all atoms $\left(\mathrm{Pb}, \mathrm{Te}, \mathrm{La}\right.$, and I), the angular momentum cut-off, $l_{\max }=3$, 
was set and semi-relativistic calculations of core level were employed. A dense mesh of $1000 k$ points in the irreducible wedge of the Brillouin-zone was used. Final converged total energy below $10^{-6}$ Ry was applied in the self-consistent cycle. The experimental doping limit $(x)$ was found to be $<0.03$ in $\mathrm{La}_{x} \mathrm{~Pb}_{1-x} \mathrm{Te}^{32}$ To realize the trend of the carrier doping effect on $E_{\mathrm{g}}$ and $m^{*}$, we calculated DOS for values of $x$ up to 0.1 for both $\mathrm{La}_{x} \mathrm{~Pb}_{1-x} \mathrm{Te}$ and $\mathrm{PbTe}_{1-x} \mathrm{I}_{x}$.

Fig. 1 shows the calculated DOS near the bottom of the conduction band $E_{\mathrm{c} \text {,bottom }}$ for $\mathrm{La}_{x} \mathrm{~Pb}_{1-x} \mathrm{Te}$ and $\mathrm{PbTe}_{1-x} \mathrm{I}_{x}$ $(0 \leq x \leq 0.1)$. The overall shape of DOS for pure-PbTe $(x=0)$ is consistent with the previous results. ${ }^{8,22,29,33}$ Apparently, there is no significant difference in DOS between pure- $\mathrm{PbTe}(x=0)$ and dilute doped-PbTe $(x=0.0001)$ for both La- and I-doped PbTe. For undoped $\mathrm{PbTe}$, strong hybridization between $\mathrm{Te}$ and $\mathrm{Pb} p$-states contributes dominantly to opening the bandgap. Interestingly, the shape of DOS of the conduction band $0.5 \mathrm{eV}$ above $E_{\mathrm{c} \text {,bottom in La- }}$ doped $\mathrm{PbTe}$ changes markedly with increasing doping concentration $x$, as shown in Fig. 1(a), which cannot be interpreted by the simple rigid-band-approximation. On the other hand, the shape of DOS of the conduction band in I-doped $\mathrm{PbTe}$ does not change dramatically within $0.6 \mathrm{eV}$ of $E_{\mathrm{c}}$, as shown in Fig. 1(b). In this case, the DOS peak of the valence band is noticeably suppressed upon I-doping. Fig. 2 shows the typical examples $(x=0.03)$ of the total and partial DOS $\left(s-, p-, d-\right.$, and $f$-states) for $\mathrm{La}_{x} \mathrm{~Pb}_{1-x} \mathrm{Te}$ and $\mathrm{PbTe}_{1-x} \mathrm{I}_{x}$. The increase in DOS might be considered as a resonant impurity, as seen in Tl- and Ti-doped PbTe. ${ }^{7,8,34,35}$ The La impurity DOS peak at high La concentration is due to $f$-states, which

(a)
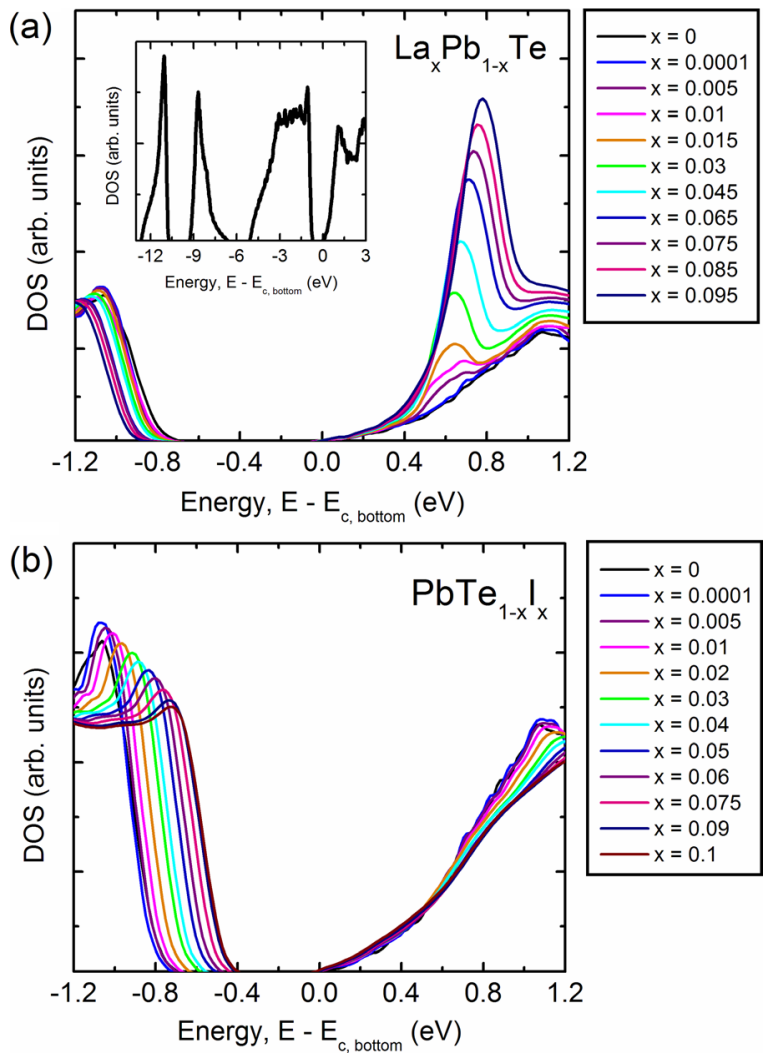

FIG. 1. Electronic DOS near the bottom of conduction band $E_{\mathrm{C}, \text { bottom }}$ for (a) La-doped $\mathrm{PbTe}\left(\mathrm{La}_{x} \mathrm{~Pb}_{1-x} \mathrm{Te}\right)$ and (b) I-doped $\mathrm{PbTe}\left(\mathrm{PbTe}_{1-x} \mathrm{I}_{x}\right)$. The inset shows the DOS of pure-PbTe $(x=0)$ with wide energy window.
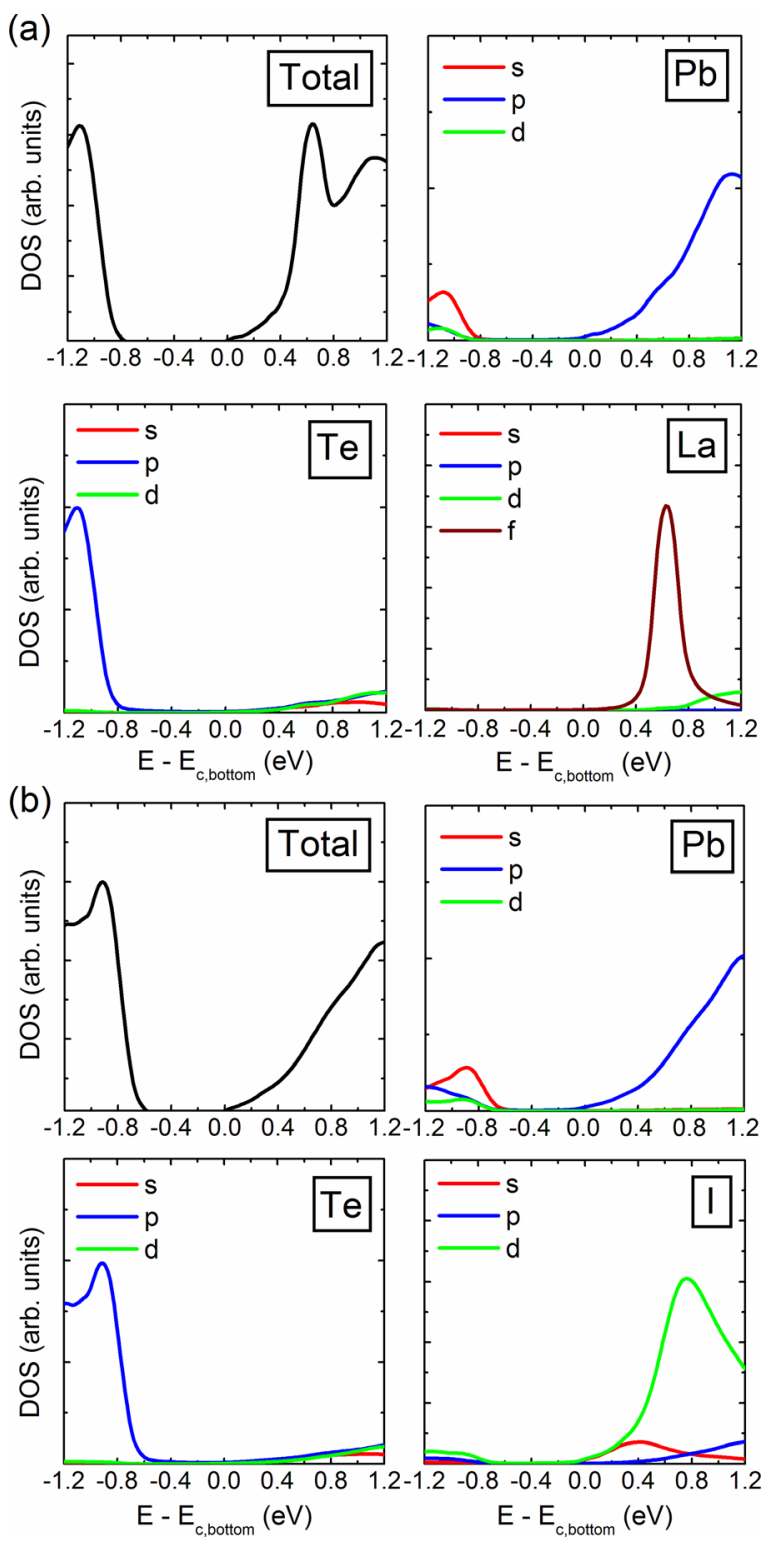

FIG. 2. Total and partial electronic DOS near the bottom of the conduction band $E_{\mathrm{C} \text {,bottom }}$ for (a) $\mathrm{La}_{x} \mathrm{~Pb}_{1-x} \mathrm{Te}(x=0.03)$ and (b) $\mathrm{PbTe}_{1-x} \mathrm{I}_{x}(x=0.03)$.

may not be delocalized sufficiently to result in resonant levels. ${ }^{8}$ However, because of the hybridization between La $f$-states (or $d$-states if $f$-states are completely localized) and $\mathrm{Pb} p$-states, the conduction band at the $L$ point can be affected by La impurity, increasing the DOS (and $m^{*}$ ) of $L$ band. While the rather broad I $d$-states also reside in the conduction band, its contribution to the total DOS is negligible. This shows excellent agreement with the experimental result, where $m^{*}$ of La-doped PbTe was found to be higher than that of I-doped $\mathrm{PbTe}$ from the measured transport properties. ${ }^{15}$ According to the Kane-band theory, ${ }^{36}$ which is often used to understand the band structure of PbTe, ${ }^{24}$ one would expect that the increase in the DOS may be also due to an increase in the bandgap. ${ }^{37}$

Another noteworthy feature is the magnitude of $E_{\mathrm{g}}$ upon doping. $E_{\mathrm{g}}$ changes continuously with varying $x$. Figure 3(a) shows $E_{\mathrm{g}}$ as a function of $x$ for La- or I-doped PbTe. The calculated value of $E_{\mathrm{g}}$ is $0.67 \mathrm{eV}$ for pure-PbTe, which is well consistent with previous reported calculation of $0.68 \mathrm{eV}$ with KKR calculation. ${ }^{22}$ Although the absolute value of the 


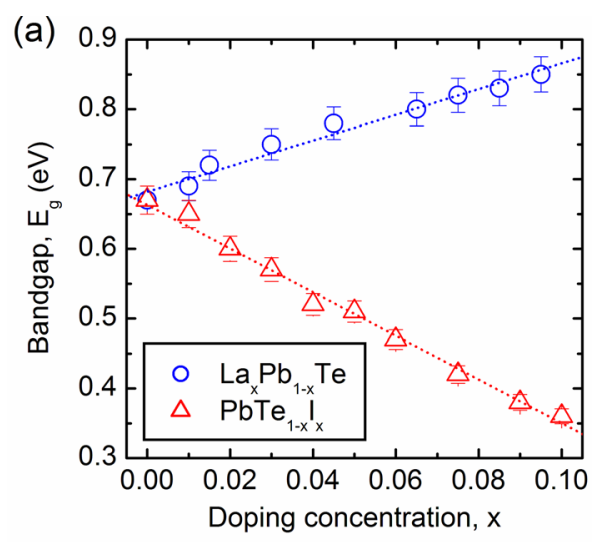

(b)

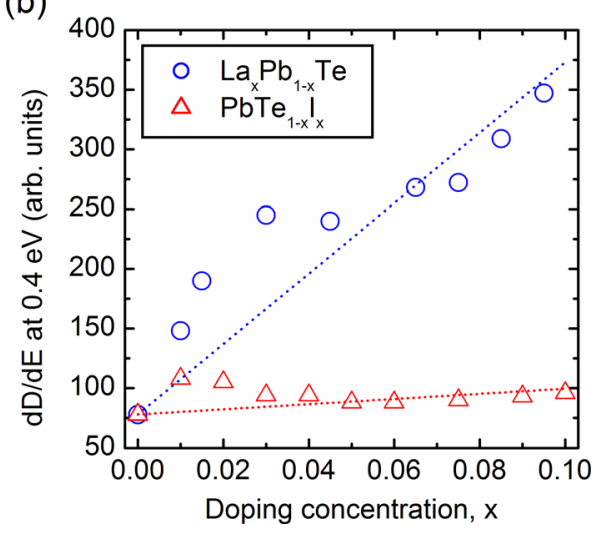

FIG. 3. (a) Bandgap and (b) $\mathrm{dD} / \mathrm{dE}$ at $E-E_{\text {c,bottom }}=0.4 \mathrm{eV}$ for (a) $\mathrm{La}_{x} \mathrm{~Pb}_{1-x} \mathrm{Te}$ and (b) $\mathrm{PbTe}_{1-x} \mathrm{I}_{x}$. The dashed lines are drawn to guide the eye.

calculated $E_{\mathrm{g}}$ is higher than the experimental result, the calculations should indicate how $E_{\mathrm{g}}$ changes with doping. One can see a distinct doping concentration dependence of $E_{\mathrm{g}}$. The $E_{\mathrm{g}}$ of La-doped $\mathrm{PbTe}$ increases with increasing $x$, while the $E_{\mathrm{g}}$ of I-doping decreases. To estimate the effective mass $m^{*}$ qualitatively, we compare the energy differential of DOS $(\mathrm{d} D / \mathrm{d} E)$ at $E-E_{\mathrm{c}, \text { bottom }}=0.4 \mathrm{eV}$, which is proportional to the effective $m^{*}$ for both La- or I-doping. The results are displayed in Fig. 3(b). For La-doped PbTe, $\mathrm{d} D / \mathrm{d} E$ increases with increasing $x$, indicating an increase in $m^{*}$ that can be presumably attribute to the hybridization between $\mathrm{La} f$-states and $\mathrm{Pb} p$-states and the increased bandgap, as discussed above. The increase in $m^{*}$ (or the decrease in $\mu$ ) through Ladoping can qualitatively explain the experimental results. ${ }^{15,25}$ On the other hand, the slope of $\mathrm{d} D / \mathrm{d} E$ versus $x$ for I-doping is rather small as compared with that of Ladoping, indicating a negligible effect on $m^{*}$ by I-doping. At the same doping level, $m^{*}$ of La-doped PbTe is higher than that of the I-doped $\mathrm{PbTe}$, which agrees qualitatively with the recent experimental results. ${ }^{15}$

In summary, we demonstrate the different effects on the electronic structure by La- or I-doping in PbTe by the KKRCPA calculation. The previously reported experimental results ${ }^{15,24,25}$ can be interpreted by the present calculation. The $m^{*}$ of La-doping is found to be higher than that of I-doping, which provides additional evidence to the experimental transport properties reported recently. ${ }^{15}$ In contrast to I-doping that a negligible effect on $m^{*}$ is found at any doping levels, La-doping shows $m^{*}$ increasing with doping concentration, which can be understood by the hybridization between La $f$-states and $\mathrm{Pb} p$-states and the increased bandgap. The present work demonstrates that KKR-CPA calculation would be a powerful tool for understanding the electronic structure of a heavily doped semiconductor, which can be used to guide the band structure engineering in both known and new thermoelectric materials.

This work is partially supported by KAKENHI No. 23760623 from JSPS and NASA-JPL.

${ }^{1}$ G. J. Snyder and E. S. Toberer, Nature Mater. 7, 105 (2008).

${ }^{2}$ G. D. Mahan and J. O. Sofo, Proc. Natl. Acad. Sci. U.S.A. 93, 7436 (1996).

${ }^{3}$ G. S. Nolas, J. L. Cohn, and G. A. Slack, Phys. Rev. B 58, 164 (1998).

${ }^{4}$ M. G. Kanatzidis, Chem. Mater. 22, 648 (2010).

${ }^{5}$ A. D. LaLonde, Y. Pei, and G. J. Snyder, Energy Environ. Sci. 4, 2090 (2011).

${ }^{6}$ Y. Pei, A. D. LaLonde, S. Iwanaga, and G. J. Snyder, Energy Environ. Sci. 4, 2085 (2011).

${ }^{7}$ J. P. Heremans, V. Jovovic, E. S. Toberer, A. Saramat, K. Kurosaki, A. Charoenphakdee, S. Yamanaka, and G. J. Snyder, Science 321, 554 (2008).

${ }^{8}$ J. P. Heremans, B. Wiendlocha, and A. M. Chamoire, Energy Environ. Sci. 5, 5510 (2012).

${ }^{9}$ A. D. LaLonde, Y. Pei, H. Wang, and G. J. Snyder, Mater. Today 14, 526 (2011).

${ }^{10}$ Y. Pei, X. Shi, A. D. LaLonde, H. Wang, L. Chen, and G. J. Snyder, Nature 473, 66 (2011).

${ }^{11}$ Y. Pei, A. D. LaLonde, N. A. Heinz, X. Shi, S. Iwanaga, H. Wang, L. Chen, and G. J. Snyder, Adv. Mater. 23, 5674 (2011).

${ }^{12}$ Q. Zhang, H. Wang, W. Liu, H. Wang, B. Yu, Q. Zhang, Z. Tian, G. Ni, S. Lee, K. Esfarjani, G. Chen, and Z. Ren, Energy Environ. Sci. 5, 5246 (2012).

${ }^{13}$ Q. Zhang, H. Wang, Q. Zhang, W. Liu, B. Yu, H. Wang, D. Wang, G. Ni, G. Chen, and Z. Ren, Nano Lett. 12, 2324 (2012).

${ }^{14}$ H. Wang, Y. Pei, A. D. LaLonde, and G. J. Snyder, Proc. Natl. Acad. Sci. U.S.A. 109, 9705 (2012).

${ }^{15}$ Y. Pei, A. D. LaLonde, H. Wang, and G. J. Snyder, Energy Environ. Sci. 5, 7963 (2012).

${ }^{16}$ Y. Pei, J. Lensch-Falk, E. S. Toberer, D. L. Medlin, and G. J. Snyder, Adv. Funct. Mater. 21, 241 (2011).

${ }^{17}$ K. F. Hsu, S. Loo, F. Guo, W. Chen, J. S. Dyck, C. Uher, T. Hogan, E. K. Polychroniadis, and M. G. Kanatzidis, Science 303, 818 (2004).

${ }^{18}$ Y. Pei, N. A. Heinz, A. D. LaLonde, and G. J. Snyder, Energy Environ. Sci. 4, 3640 (2011).

${ }^{19}$ E. S. Toberer, A. F. May, and G. J. Snyder, Chem. Mater. 22, 624 (2010).

${ }^{20}$ A. F. May, D. J. Singh, and G. J. Snyder, Phys. Rev. B 79, 153101 (2009).

${ }^{21}$ J. D. König, M. D. Nielsen, Yi -Bin Gao, M. Winkler, A. Jacquot, H. Böttner, and J. P. Heremans, Phys. Rev. B 84, 205126 (2011).

${ }^{22}$ C. M. Jaworski, J. Tobola, E. M. Levin, K. Schmidt-Rohr, and J. P. Heremans, Phys. Rev. B 80, 125208 (2009).

${ }^{23}$ D. J. Singh, Phys. Rev. B 81, 195217 (2010).

${ }^{24}$ Y. I. Ravich, B. A. Efimova, and I. A. Smirnov, Semiconducting Lead Chalcogenides (Plenum, New York, 1970).

${ }^{25}$ G. T. Alekseeva, M. V. Vedernikov, E. A. Gurieva, P. P. Konstantinov, L. V. Prokof'eva, and Yu. I. Ravich, Semiconductors 32, 716 (1998).

${ }^{26}$ H. Akai, J. Phys. Soc. Jpn. 51, 468 (1982).

${ }^{27}$ H. Akai, J. Phys.: Condens. Matter 1, 8045 (1989).

${ }^{28}$ M. Schröter, H. Ebert, H. Akai, P. Entel, E. Hoffmann, and G. G. Reddy, Phys. Rev. B 52, 188 (1995).

${ }^{29}$ C. M. Jaworski, B. Wiendlocha, V. Jovovic, and J. P. Heremans, Energy Environ. Sci. 4, 4155 (2011).

${ }^{30}$ R. Dalven, Infrared Phys. Technol. 9, 141 (1969).

${ }^{31}$ U. von Barth and L. Hedin, J. Phys. C 5, 1629 (1972).

${ }^{32}$ Z. A. Iskender Zade, C. I. Abilov, V. D. Faradzhev, E. Z. Zeinalov, and E. M. Kurbanov, Inorg. Mater. 26, 366 (1990).

${ }^{33}$ E. A. Albanesi, C. M. I. Okoye, C. O. Rodriguez, E. L. Peltzer y Blanca, and A. G. Petukhov, Phys. Rev. B 61, 16589 (2000).

${ }^{34}$ M. N. Vinogradova, E. A. Gurieva, V. I. Zharskii, S. V. Zarubo, and L. V. Prokofeva, Sov. Phys. Semicond. 12, 387 (1978).

${ }^{35}$ F. Sizov, V. Teterkin, L. Prokofeva, and E. Gurieva, Sov. Phys. Semicond. 14, 1063 (1980)

${ }^{36}$ E. Kane, J. Phys. Chem. Solids. 1, 249 (1957).

${ }^{37}$ I. A. Smirnov, B. Y. Moizhes, and E. D. Nensberg, Sov. Phys. Solid State. 2, 1793 (1961). 